\title{
À la recherche du schwa : données, méthodes et théories
}

\author{
Durand, Jacques \\ CLLE-ERSS (UMR5263), Université de Toulouse II, CNRS et IUF \\ jacques.durand@univ-tlse2.fr
}

\section{Introduction}

Choisir le schwa comme objet d'étude, c'est d'emblée se situer dans une filiation de travaux très nombreux, trop nombreux pour certains, qui cherchent à cerner un objet fugace, hybride voire absent comme en témoignent des étiquettes concurrentes comme 'e caduc', 'e instable', 'e féminin', 'e sourd' ou 'e muet'. 'Un mot sur la terminologie choisie ici. Le terme de 'schwa' n'est pas nouveau en grammaire française puisque Arnauld et Lancelot dans leur Grammaire générale et raisonnée (1660) y renvoient dans leur chapitre premier où ils démontrent qu'ils ne confondent absolument pas les sons (pour lequels ils utilisent aussi le terme classique de 'lettre') et les caractères orthographiques qui les représentent. À côté des neufs sons simples qu'ils faut reconnaître selon eux en français $a, \dot{e}, \dot{e}, i, o, o ́, e u, o u, u$ (API /a $\varepsilon$ e i $ว$ o $\varnothing \mathrm{u}$ y/), il existe un élément supplémentaire :

«Il reste l'e muet ou féminin, qui n'est dans son origine qu'un son sourd, conjoint aux consonnes lorsqu'on les veut prononcer sans voyelle [...] ce que les Hébreux appellent scheva, surtout lorsqu'il commence la syllabe. Et ce scheva se trouve nécessairement en toutes les langues, quoiqu'on n'y prenne pas garde, parce qu'il n'y a point de caractère pour le marquer. Mais quelques langues vulgaires, comme l'allemand ou le français, l'ont marqué par la voyelle $e$, ajoutant ce son aux autres qu'elles avaient déjà : et de plus ils ont fait que cet $e$ féminin fait une syllabe avec sa consonne, comme est la seconde dans netteté, j'aimerai, donnerai, ce que ne faisait pas le scheva dans les autres langues, quoique plusieurs fassent cette faute en prononçant le scheva des Hébreux. Et ce qui est encore plus remarquable, c'est que cet $e$ muet fait souvent tout seul en français une syllabe, ou plutôt une semisyllabe, comme vie, vue, aimée. » (Arnauld et Lancelot, $1660: 10-11$ ).

Cette citation, qui mériterait une véritable exégèse, montre bien que, depuis fort longtemps, le schwa fait problème phonétiquement et phonologiquement, comme nous le dirions aujourd'hui. C'est la raison pour laquelle je ne chercherai pas à défendre l'étiquette choisie. Schwa sera pour moi un terme préthéorique et je ne m'interdirai pas non plus d'utiliser les termes «e muet» ou «e caduc » lorsque c'est l'usage des auteurs que je citerai, car tous ces termes nous orientent vers un ensemble de phénomènes qui, dans la diversité des pratiques, ne forment pas un tout cohérent. Et précisément parce que ce tout n'est pas cohérent et pour mieux expliquer ma propre posture, on me pardonnera de commencer par quelques brefs rappels historiques. Comme le dit si justement Bourdieu : «Comprendre, c'est comprendre le champ avec lequel et contre lequel on s'est fait »(2004:15).

Après une esquisse rapide de positions diverses sur le schwa, nous nous pencherons sur la question des données et survolerons le cadre méthodologique dans lequel nous travaillons, celui du programme PFC (Phonologie du français contemporain : usages, variétés et structure) $)^{2}$. Nous essayerons de démontrer ensuite comment un renouvellement et un élargissement des données permettent de mieux cerner certains 
problèmes. En parlant du schwa dans diverses variétés dont le français méridional ou le français de Suisse, nous défendrons une approche de corpus où se côtoient analyses phonologiques et phonétiques. Si nous ne parcourrons pas tous les espaces ouverts par nos prédecesseurs, nous espérons que certains chemins que nous emprunterons nous amèneront vers un tout petit peu plus de lumière. ${ }^{3}$

\section{Du structuralisme au générativisme}

La trame historique des travaux autour du schwa est si complexe qu'on me pardonnera d'entamer ce bref regard rétrospectif par Martinet, héritier de Trubetzkoy et de l'école de Prague, et fondateur de la phonologie structurale en France. Un des soucis majeurs de Martinet est de désintriquer la phonologie de l'usage orthographique. Dans 'Qu'est-ce que le «e muet »' (ch. 13 de Martinet 1969), il insiste sur le fait que dans l'usage parisien, $-e$ n'a laissé aucune trace à la finale des mots : "patte se prononce comme pat, messe comme mess, coque comme coq, boxe comme box.» Il note aussi que pour divers groupes consonantiques complexes en finale de mot, la détente vocalique qu'on peut parfois entendre et qu'on pourrait vouloir transcrire comme une voyelle centrale n'est pas phonologique puisqu'elle est aussi bien présente dans Budapest que dans peste, par exemple, et n'est donc pas à porter au crédit de «e muet » (1969 : 212). En position interne, dans le contexte potentiel VCəCV, il n'y a pas de trace non plus et il n'existe aucune différence entre calepin et alpin ou allemand et calmant. Il ajoute : «Il est certain, que lorsqu'un mot est plus souvent lu que prononcé, le $e$ muet peut influencer le débit : quiconque ne connait Montereau que par les livres d'histoire tendra à reproduire le mot comme il le ferait d'une succession monte rôt. Mais ceux qui connaissent bien les abords de la Seine en amont de Paris ne prononcent pas le nom de cette ville autrement que mon trot /mõ tro/.» (1969: 213). Pour Martinet, la voyelle qui apparait dans de nombreuses séquences (ourse blanc à côté de livre blanc) n'est pas un phonème mais un simple «lubrifiant»dont la présence est déterminée par la fameuse loi des trois consonnes qu'avait exploré Grammont (1894). Mais que penser du 'e' muet dans les syllabes initiales de mot ?

La position de Martinet est que la présence ou l'absence d'une voyelle dans une des deux premières syllabes d'un mot comme relever ne nous autorise nullement à y poser un phonème. Il approuve le grammairien du XVIII ${ }^{\mathrm{e}}$ siècle Gile Vaudelin dont il décrit ailleurs le système et qui proposait de transcrire releva / $\mathrm{rlva} /$ et brebis / brbi/. Le fait que beaucoup de locuteurs prononcent un mot comme pelote avec un $/ œ /$ ou un $/ ø /$, en alternance avec zéro, dans la première syllabe nous interdit de faire l'hypothèse d'un véritable phonème «clignotant ». Comme le dit Walter $(1976: 288)$ dans sa synthèse fonctionnaliste sur le sujet: «la question du statut phonologique du e muet ne se pose que pour ceux qui ne confondent sa réalisation ni avec le phonème /œ/ ni avec le phonème /ø/. » Autrement dit, seuls les locuteurs qui ont un vrai schwa phonétique dans cette position (une voyelle centrale non-arrondie [ə]) distinct de ces deux voyelles possèdent un phonème supplémentaire /ə/. Pour les autres, il y a des /œ/ et des /ø/ stables ou instables (par exemple peupler face à pelote).

Dès ses premiers travaux, la phonologie générative classique a relancé le débat en posant pour le français de référence une différence au niveau sous-jacent entre mots se terminant par consonne(s) + schwa et mots à finale consonantique (cf. Schane 1968a,b, Dell 1973a,b). En effet, la liaison s'explique dans ce cadre par la présence d'une consonne latente qui est effacée devant consonne ou à la pause. Par exemple, petit animal se voit attribuer la forme sous-jacente /pətit\#animal/ et la prononciation [poetitanimal] ${ }^{4}$ découle naturellement de la forme de départ modulo l'effacement de la frontière de mot et l'ajustement phonétique de la qualité du schwa de la première syllabe. En revanche, dans petit cadeau /patit\#kado/, le /t/ latent devra être effacé. Les mots morphologiquement féminins et diverses formes verbales ont tous un 
schwa «abstrait » qui protège la consonne finale en la dérobant à la règle d'effacement (petite allée = /pə titə\#ale/). En français standard, ce schwa est effacé mais seulement après l'application de la règle de troncation pour produire, en conjonction avec d'autres règles tardives, les formes de surface requises (par exemple, petite allée $=[\mathrm{ptit} \# \mathrm{ale}])$. Pour justifier une telle analyse, des chercheurs comme Schane, Dell ou Selkirk, pour ne citer qu'eux, invoquent à côté des généralisations établies le fait que les schwas abstraits postulés se retrouvent dans certains styles oratoires (la poésie, par exemple) ou dans la chanson. L'orthographe française, vue comme diabolique par les tenants de la transcription phonémique, se voit également justifiée comme reflétant des régularités plus profondes d'ordre morphophonologique ou phonosyntaxique. Enfin, la variation dialectale trouve aussi sa place dans la réflexion puisque le français méridional est pris comme preuve vivante de la réalité de ces schwas sous-jacents. La différence entre un accent du Midi et un accent du nord de la Loire ne tiendrait alors qu'à des ajustements proches de la surface. L'accent standard nécessite tout simplement un plus grand nombre de règles d'effacement que l'accent du Midi mais tous les deux partagent les mêmes formes profondes.

Ces analyses initiales dans le cadre génératif ont lancé une foule d'études sur le sujet du schwa avec des reformulations dans les divers modèles qui se sont succédé. Très tôt, des analyses plus concrètes que les travaux fondateurs ont été proposées (par exemple Morin 1978, Lyche 1978). Diverses études ont souligné que la graphie (<e> en syllabe orthographique ouverte) ne suffisait pas et que de nombreux schwas putatifs correspondaient à des voyelles stables ou stabilisées. D'un autre côté, les développements de la phonologie multilinéaire ou non linéaire ont permis de modéliser plus élégamment les patrons de présence / absence. Ainsi à la suite d'Anderson (1982), plusieurs travaux ont exploré le concept de voyelle phonologique sous-spécifiée ou non-spécifiée qui peut être absente ou recevoir une interprétation phonétique quand diverses conditions syllabiques sont remplies (par exemple, Durand 1986, Tranel 1987). Par ailleurs, le concept métrique de pied a permis l'exploration du schwa dans une position faible (Durand 1976, Selkirk 1978). Plus récemment, c'est la théorie de l'optimalité qui a été mise à contribution dans des variantes qui ne sont pas nécessairement compatibles entre elles (Tranel 2000, Eychenne 2006). Ainsi Tranel (2000) propose-t-il que le caractère facultatif de l'effacement de schwa est dû au rang variable d'une consonne flottante ECONOMIE DE SYLLABE au sein de la hiérarchie de contraintes. En revanche, Côté $(2000,2008)$ rejette l'approche syllabique et favorise une description qui se fonde sur la saillance perceptuelle des consonnes ou des groupes de consonnes qui délimitent les positions à schwa. Comme pour tout problème complexe, il n'émerge pas une solution à laquelle tout le monde se rallie. Un des problèmes auxquels nous sommes cependant confrontés depuis le début est la nature des données dans leur variabilité. C'est la question qui nous préoccupera dans le reste de ce travail.

\section{Les données et leur analyse}

Nous avons entamé notre bref survol en partant de la position de Martinet. Ce dernier mentionne souvent son propre usage, mais est engagé dans une perspective d'élargissement de la base empirique de la phonologie. On se souviendra que son ouvrage de 1945, La prononciation du français contemporain, a pour sous-titre en première page intérieure Témoignages recueillis en 1941 dans un camp d'officiers prisonniers. Il faut également mentionner le travail de préparation pour le Dictionnaire de la prononciation française dans son usage réel publié en 1973 avec Walter. Cependant ni la réponse à des questionnaires comme ceux de 1941, ni la lecture à haute voix de phrases contenant les mots cibles du dictionnaire de 1973, ne semblent suffisant pour observer finement un espace aussi complexe que celui que dessine le schwa. Il est douteux, par exemple, que la réponse à une question comme la suivante soit vraiment instructive sur ce que font effectivement les locuteurs : "Quelle prononciation vous paraît la 
plus naturelle: a) de j'me dis, je m'dis, je me dis? b) de j'me le d'mande, je m'le demande, j'me le d'mande, je me le demande » (Martinet $1945: 53$ ). Si on veut vérifier rigoureusement l'application de la «loi des trois consonnes», qui est l'objectif explicite de cette question, l'analyse de données conversationnelles semble incontournable.

A l'opposé de ce travail, la phonologie générative est prise en tenaille dans un double mouvement. Schane (1968a) a bien lu ses classiques, à la fois en synchronie et en diachronie, mais s'appuie sur une description standard dont les principaux défenseurs dans sa bibliographie sont Delattre (1951), Fouché (1956) et Grevisse (1964). C'est donc la norme qu'il retrouve, soit par les instructions que fournissent ces travaux à des étrangers désireux de maîtriser la langue française, soit par les conseils donnés aux francophones soucieux de respecter le bon usage. Lorsqu'on invoque la norme on retrouve inévitablement l'orthographe et une partie de la diachronie. Ces biais pèsent lourd sur les traitements génératifs classiques et la tradition qu'ils inaugurent. A l'opposé de Schane, Dell (1973a: 195) dans son étude fouillée du schwa informe le lecteur qu'il décrit son propre parler et ajoute en note : «Né en 1943, a vécu dans un petit village de l'Yonne jusqu'en 1949, et réside à Paris depuis. » Dell est un observateur méthodique, sait mettre en parallèle ses observations et celles de travaux plus classiques et est souvent attentif à des variations au sein de son propre usage. Son approche dans le cadre chomskyen est cohérente. Si l'objet est le langage-I (I-language), dans la terminologie chomskyenne, à savoir un système individuel, intensionnel et interne, et si l'intuition (au sens de jugement de bonne formation) est la meilleure voie d'accès aux données, alors la description par le linguiste de son propre système est incontournable. Sans nous appesantir sur la question, rappelons néanmoins qu'en essayant de décrire ses propres pratiques on est inconsciemment travaillé par les conflits divers qui animent la vie sociale d'une langue. D'autre part, les arguments en faveur du rôle de l'intuition dans le domaine syntaxique ont toujours tourné sur les potentialités infinies qu'offre la récursion mais cette dernière est très controversée en phonologie. ${ }^{5}$ Il est d'ailleurs significatif que Fitch, Hauser et Chomsky (2005) dans leur mise au point sur l'évolution du langage excluent la phonologie de la Faculté de langage au sens étroit (LFN, Language Faculty Narrow). Le travail sur corpus ainsi que le travail expérimental que défendent phonéticiens et psycholinguistiques semblent donc nécessaires (Laks 2008, Durand 2009).

Je ne reviendrai pas ici en détail sur le cadre méthodologique que nous défendons dans le programme PFC (Phonologie du français contemporain : usages, variétés et structure) actuellement coordonné par Marie-Hélène Côté, Bernard Laks, Chantal Lyche et moi-même. Une cinquantaine de chercheurs, étudiants inclus, nous accompagnent dans cette démarche collective. Nous préférons désormais le terme de 'programme' à celui de 'projet' car les recherches entreprises sont ouvertes : il y a divers sousprogrammes originaux, des extensions diverses et une pluralité d'outils formels et computationnels (voir la note 2). Au coeur de notre démarche et en laissant de côté les extensions en cours, nous avons placé un large panel d'enquêtes de modeste taille mais privilégiant en revanche une vaste couverture géographique. Dans chaque point d'enquête nous travaillons dans des réseaux denses comprenant plusieurs générations de locuteurs et un nombre aussi égal que possible d'hommes et de femmes. Quatre enregistrements sont au centre du dispositif : la lecture à haute voix d'une liste de mots, celle d'un texte, une conversation semi-dirigée et une conversation libre où nous laissons des intimes entre eux pour nous rapprocher asymptotiquement du vernaculaire. Les conversations sont évidemment notre cible privilégiée et les données que je présenterai (sauf mention explicite contraire) seront tirées des conversations. La lecture à haute voix est néanmoins d'une grande utilité et permet une description fine de divers aspects du rapport graphie-phonie qui est si centrale dans une langue à vieille tradition orthographique comme le français. Les enregistrements sont en partie transcrits orthographiquement avec alignement au signal et soumis à plusieurs types de codages. Divers outils génériques ou propres au projet permettent d'explorer 
les données et de les croiser avec des métadonnées comme l'âge de la personne ou sa profession par exemple. Nous avons consacré beaucoup d'énergie à mettre au point et expliciter nos codages. C'est le prix à payer si une base de données peut être consultée par d'autres chercheurs que les membres d'un programme et si son exploration permet d'étayer ou d'invalider des observations et des hypothèses déjà formulées par d'autres chercheurs. Les cycles de conjectures et de réfutations qui depuis Popper (1963) constituent le coeur de la démarche scientifique s'appliquent aussi à des premiers niveaux d'observation et d'analyse et pas seulement aux systèmes formels parachevés qui seuls retiennent l'attention de certains linguistes.

\section{Le schwa en français septentrional et méridional}

Nous avons signalé plus haut que les analyses dans la tradition générative ont souvent posé pour le français désormais dit de référence une opposition entre schwa et zéro en position finale, dans le cas du féminin, de la conjugaison verbale et pour des oppositions lexicales comme laque et lac ou phare et fard traités par Martinet et bien d'autres comme identiques en fait et en droit. Si c'était la position de Dell dans la première édition de son fameux ouvrage Les règles et les sons (1973), il revient dans la deuxième version de ce même ouvrage à la solution qu'il avait adoptée dans sa thèse de doctorat où il traitait toutes les consonnes finales réalisées comme des attaques de syllabe à voyelle vide ${ }^{6}$ (schwa) : «Pacte et tact ont (à la consonne initiale près) des représentations phonologiques identiques, et la distinction entre les deux classes de mot est de nature purement orthographique. » (1985: 236, note 39). Je me contenterai d'affirmer qu'une telle décision ébranle profondément la défense de l'orthographe montée par Schane $(1968 \mathrm{a}, \mathrm{b})$ et Dell $(1973 \mathrm{a}, \mathrm{b})$ dans leurs travaux fondateurs.

De fait, les enquêtes PFC effectuées dans la partie septentrionale de l'hexagone ne justifient pas l'existence d'une opposition schwa / zéro à la finale, que ce soit devant un autre mot avec une attaque consonantique (belle partie) ou dans un contexte de pause (Elle est belle !). Ce qu'elles confirment, en revanche, c'est que, depuis plus de deux décennies, de nombreux locuteurs utilisent très fréquemment un écho vocalique (un schwa phonétique) après consonne dans des contextes où les travaux génératifs classiques postulaient une règle obligatoire d'effacement de schwa phonologique du type :

(1) $/ \partial /-->\varnothing /-\#\{\#, C\}$

Anita Berit Hansen qui étudie ce phénomène depuis une bonne vingtaine d'années a récemment exploré cette question dans le cadre PFC avec de jeunes Parisiens et note que cette « voyelle d'appui », comme l'appelle Candea (2000), apparaît aussi bien pour des mots qui se terminent par un $<\mathrm{e}>$ orthographique que pour des mots qui se terminent par une consonne orthographique phonétiquement présente. C'est bien sûr le cas de l'expression Bonjour-e ! qui sert d'indexation à ce phénomène chez les linguistes. Hansen (2013 : 165-167) note que, même dans la lecture à haute voix, on peut observer l'apparition de ce schwa non étymologique puisque certains lecteurs produisent ce schwa d'appui dans la séquence son vin blanc sec-[ə] au sein du texte PFC

La présence statistiquement significative de schwas d'appui rend illusoire l'idée qu'il y aurait une opposition phonologique en français septentrional entre présence et absence de schwa final. Ceux qui invoquent la différence dans leur « dialecte » entre un tel hameau (schwa jugé impossible après tel) et une telle housse (schwa jugé possible ou préféré dans telle) font porter bien de poids à des contextes impliquant le $h$ aspiré, contextes qui sont directement liés à la distinction sociale et au capital symbolique 
des agents linguistiques. Or ces derniers sont intimement liés à la compétence orthographique ce qui ne signifie pas que les locuteurs intègrent toujours de façon régulière ses effets. Ainsi, lorsque Pierre Encrevé (1988), à qui nous devons tant en sociophonologie, propose une représentation phonologique différente pour les mots morphologiquement féminins du type $<\mathrm{XCe}>$ et les mots du type $<\mathrm{XC}>$ correspondants (ourse vs. ours, patte vs. pat, nette vs. net), il ne fournit de fait qu'un seul argument «phonétique» qui repose cependant sur un contexte métalinguistique où de nombreux locuteurs graphématisés consultent leur lexique orthographique. Il déclare : «Nous faisons ce choix sur l'argument, peut-être pas décisif mais non dénué de poids, que, en dépit du fait que l'énoncé [ursəblø] ne permet pas de reconnaître s'il s'agit du masculin l'ours bleu ou du féminin l'ourse bleue, si l'on dit non pas l'ours [urs] mais l'ourse [ursə], tout francophone comprendra que le [ə] désigne le féminin. C'est donc que quelque chose différencie dans la structure sous-jacente ours et ourse quant à la probabilité de l'épenthèse d'un schwa dans le contexte de la pause où elle est normalement exclue, distinction qui nous paraît bien traduite par l'opposition entre vraie épenthèse (celle qui peut affecter le masculin ours) et fausse épenthèse (celle qui peut affecter le féminin ourse).» (p. 228-229). Si on doit se fier à l'intuition, j'ai quant à moi un sentiment différent sur la capacité des locuteurs septentrionaux à percevoir l'opposition entre schwa et zéro en finale de mot qui caractérise l'accent méridional conservateur qui est le mien. En effet, lorsque je me suis trouvé dans des situations où, incertain de ce qui avait été dit, j'ai produit des

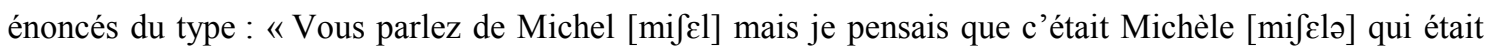
responsable? », ou inversement, «Vous parlez de Michèle [mifelə] mais je pensais que c'était Michel [mifel] qui était responsable? ", j'ai pu constater que mes interlocuteurs septentrionaux semblaient pour ainsi dire « sourds » à la distinction entre un schwa phonétique qui peut-être léger et son absence. Par ailleurs, j'ai pu constater quasiment a contrario qu'un 'e muet' orthographique réalisé phonétiquement dans une position inattendue pour un locuteur septentrional pouvait provoquer une incompréhension totale. Me rendant il y a quelques années de Paris à Abbeville, j'ai eu le malheur de demander à un passant si j'étais bien sur la route pour [abəvila]. Après plusieurs échecs de compréhension, j'ai compris (bien lentement, il est vrai pour un linguiste !) que ma prononciation était peut-être à incriminer. En tout cas, en adoptant la prononciation [abvil], j'ai immédiatement reçu l'information qu'en poursuivant mon chemin je n'étais qu'à quelques kilomètres de cette cité. Je note enfin que dans diverses enquêtes PFC sur le français septentrional, la présence de «e muets » dans la graphie n'a aucune influence sur la réalisation de schwas en lecture à haute voix. Au contraire, pour les locuteurs méridionaux de nos enquêtes la présence de «e muets » orthographiques déclenchent plus de schwas en lecture que dans les conversations. Les deux systèmes septentrionaux et méridionaux sont loin d'être isomorphes dans l'oral spontané et dans l'oralisation de l'écrit.

Cependant, qu'en est-il vraiment de la prononciation méridionale dans la diversité des usages ? Je ne prétendrai pas fournir une image globale de la situation dans tout le sud de la France. Je m'appuierai ici sur des enquêtes PFC qui ont été étudiées dans le cadre de notre programme. Nous examinerons plus bas deux enquêtes effectuées dans des villages languedociens (Douzens, 600 habitants, et Lacaune, 300 habitants), deux enquêtes qui se sont déroulées à Aix-Marseille et Marseille, et une enquête à Biarritz au Pays Basque. Nous examinerons ensuite des travaux en cours sur Toulouse qui vise à constituer une base de données plus conséquente que les précédentes avec 120 témoins (dont une soixantaine déjà enregistrés).

La première chose à signaler est que l'opposition schwa / zéro en finale de mot est structurellement bien attestée et qu'elle correspond largement à la présence d'un $<\mathrm{e}>$ orthographique. Nos codages partent de la tanscription orthographique de nos enregistrements et examinent à la finale tous les mots se terminant soit par $<\mathrm{e}>$, soit par une consonne orthographique correspondant à une consonne phonétique (par exemple, 
laque et $l a c$ ). Ce qu'on peut observer est une asymétrie entre les mots du type lac et les mots du type laque. Dans nos résultats jusqu'ici, alors que les mots du type lac sont massivement réalisés avec une consonne finale sans suite vocalique, les mots comme laque alternent entre présence et absence d'une voyelle finale que nous traiterons ici comme un schwa phonologique. Ce n'est pas sa qualité phonétique qui compte car elle varie énormément mais le fait qu'elle est prosodiquement faible et s'efface normalement dans un groupe rythmique soudé lorsque le mot suivant commence par une voyelle (par exemple, tell(e) entreprise). Nous allons voir que dans cette position de grands changements sont en cours qui affectent différentiellement les locuteurs selon leur cantonnement sociolinguistique. Mais tout d'abord nous ferons quelques rapides remarques sur le schwa en position interne de mot. Pour la prononciation septentrionale, de nombreux chercheurs posent des schwas sous-jacents dans des mots comme tell(e)ment (contexte que nous symboliserons par $\mathrm{VCeC}$ ) par parallélisme avec d'autres mots comme autrenent où l'environnement consonantique force le maintien. Dans les accents méridionaux conservateurs, la voyelle médiane dans ces mots ne chute pas mais on peut construire un argument pour la traiter comme un schwa à partir de critères morphologiques (tellement $=$ telle + ment $)$ et phonologiques (la voyelle médiane est prosodiquement faible et la voyelle à gauche suit la loi de position). Néanmoins, il y a des prononciations qui ne correspondent pas exactement à ce patron : par exemple celle du mot médecin s'il est prononcé [medøsen] de façon identique à mes deux seins.

En nous appuyant sur les données et les analyses présentées par Durand, Eychenne et Lyche (2013), comparons maintenant pour les cinq enquêtes que nous avons choisies les effacements dans le contexte VCe\#C (par ex. belle table) avec les effacements en position interne de mot (VCeC). Le Tableau 1 cidessous révèle une très grande différence entre ces deux contextes. La notation $\mathrm{n} 1 / \mathrm{n} 2$ fournie entre parenthèses après le pourcentage de schwas réalisés désigne le nombre de schwas présents rapporté au nombre total de positions attestées. Par conséquent, 94/99 à Douzens pour VCeC signifie qu'il y avait 99 mots de type tellement et que dans 94 cas le schwa interne est prononcé.

\begin{tabular}{|l|l|l|}
\hline & $\begin{array}{l}\text { Position interne } \\
\text { VCeC }\end{array}$ & $\begin{array}{l}\text { Position finale } \\
\text { VCe\#C }\end{array}$ \\
\hline & Schwa présent & Schwa présent \\
\hline Douzens & $94.9 \%(94 / 99)$ & $78.7 \%(420 / 533)$ \\
\hline Lacaune & $97 \%(63 / 65)$ & $78.8 \%(328 / 416)$ \\
\hline Aix-Marseille & $74.5 \%(44 / 59)$ & $19 \%(44 / 232)$ \\
\hline Marseille & $65.2 \%(62 / 95)$ & $17.3 \%(62 / 357)$ \\
\hline Biarritz & $71.8 \%(97 / 135)$ & $26.4 \%(168 / 636)$ \\
\hline
\end{tabular}

Tableau 1. Présence de schwas en position interne et finale: comparaison de données conversationnelles tirées de cinq enquêtes effectuées dans le sud de la France. 
Le Tableau 1 révèle à l'évidence une grande différence entre Douzens et Lacaune d'une part et les villes de l'autre. La position interne est cependant moins propice à l'effacement et le changement semble être dans sa phase initiale. Néanmoins, en ce qui concerne le schwa, ces observations confirment qu'il existe une tendance au nivellement vers l'usage septentrional dans les villes du sud de la France. Cette conclusion est confortée par l'observation que ce sont les jeunes locuteurs qui constituent le fer de lance du changement comme le démontre le Tableau 2 établi à partir des mêmes cinq points d'enquête:

\begin{tabular}{|l|l|l|}
\hline & $\begin{array}{l}\text { Position interne } \\
\text { VCeC }\end{array}$ & Position finale \\
Age & Schwa présent & Schwa présent \\
\hline$<26$ & $21.2 \%(17 / 80)$ & $31.4 \%(115 / 366)$ \\
\hline $26-59$ & $47.8 \%(180 / 376)$ & $21.7 \%(189 / 871)$ \\
\hline$>59$ & $66.9 \%(249 / 372)$ & $78.7 \%(811 / 1030)$ \\
\hline
\end{tabular}

Tableau 2. Présence de schwas en position interne et finale: comparaison en fonction de l'âge de données conversationnelles tirées de cinq enquêtes effectuées dans le sud de la France

Dans ce deuxième tableau nous constatons que les plus jeunes sont les plus innovateurs sauf pour la position finale où les moyens sont plus en avance. $\mathrm{Si}$, pour la position finale, nous modifions les groupes dâge et les restreignons à deux, à savoir au-desous et au-dessus de 40 ans, on peut établir une différence majeure: le taux de présence de schwa pour les locuteurs de moins de 40 ans chute à $30.3 \%$, alors qu'il est de $67.4 \%$ pour les locuteurs de plus de 40 ans. Cela indiquerait que le changement a déjà pris place sur deux générations.

Les résultats ci-dessus sont confirmés dans l'enquête LVTI (Langue Ville Travail Identité) en cours à Toulouse pour laquelle 21 locuteurs ont d'ores et déjà pu être analysés suivant le protocole PFC. ${ }^{7}$ Le Tableau 3 ci-dessous résume les observations déjà disponibles. La position initiale ${ }^{8}$ qui est incluse ici retiendra notre attention plus loin.

\begin{tabular}{|l|l|l|l|l|}
\hline & $\begin{array}{l}\text { Position initiale : } \\
\text { V\#CeC }\end{array}$ & $\begin{array}{l}\text { Position interne : } \\
\text { VCeC }\end{array}$ & $\begin{array}{l}\text { Position finale : } \\
\text { VCe\#C }\end{array}$ & Total \\
\hline $20-23$ ans & $68 \%(65 / 95)$ & $17 \%(14 / 84)$ & $10 \%(71 / 732)$ & $16 \%(150 / 911)$ \\
\hline $27-35$ ans & $72 \%(31 / 43)$ & $21 \%(7 / 34)$ & $15 \%(72 / 492)$ & $19 \%(110 / 569)$ \\
\hline $53-67$ ans & $93 \%(40 / 43)$ & $86 \%(61 / 71)$ & $44 \%(197 / 448)$ & $53 \%(298 / 562)$ \\
\hline $79-95$ ans & $91 \%(20 / 22)$ & $95 \%(21 / 22)$ & $56 \%(89 / 160)$ & $64 \%(130 / 204)$ \\
\hline Total & $77 \%(156 / 203)$ & $49 \%(103 / 211)$ & $23 \%(429 / 1832)$ & $31 \%(688 / 2246)$ \\
\hline
\end{tabular}

Tableau 3. Présence de schwas en position initiale, interne et finale: comparaison en fonction de l'âge de données conversationnelles de l'enquête de Toulouse.

Quelle que soit la position du schwa au sein des mots, les plus jeunes montrent une baisse significative du taux de réalisation. Cette assymétrie est particulièrement visible si l'on place une frontière vers 40 ans $(\mathrm{p}<0.05$, test $\chi 2)$. On notera que, globalement, la position finale est la plus touchée par l'affaiblissement 
du schwa, la position interne offre une image contrastée et la position initiale montre un fort taux de réalisation.

Un travail attentif sur les données de Toulouse démontre que les mots du type lac ne se comportent pas comme les mots du type laque. De plus, l'influence de la graphie peut-être observée entre la tâche de lecture à voix haute et les conversations : le taux de réalisation d'un schwa dans la lecture du texte PFC est significativement plus élevé que dans les conversations (respectivement $60 \%$ et $51 \%$ de réalisations effectives). Toutefois, nous devons signaler que deux locuteurs sur 21 (âgés de 23 et 29 ans) réalisent occasionnellement un schwa derrière une consonne orthographique finale en position pré-consonantique ou pré-pausale. L'opposition entre des mots tels que «mal» et «malle» est donc menacée et on peut s'interroger sur le degré de nivellement de tels locuteurs en ce qui concerne le schwa. Le système qu'ils utilisent semble profondément instable et on peut faire le pari qu'en élargissant nos bases de données, on trouvera de plus en plus de jeunes locuteurs qui ont basculé vers un système septentrional en finale de mot.

Revenons maintenant sur la question du schwa dans les syllabes initiales de mot (ex. semaine). Dans ce contexte, nos codages révèlent un fort taux de réalisation dans tous les points d'enquête méridionaux. À partir des données d'Aix-Marseille et de Marseille, Coquillon et Turcsan (2013) établissent un taux de présence global de $91 \%$ dans cette position et montrent que seuls quelques mots fréquents sont impliqués (par exemple, petit, serai, semaine). Ils concluent : «Our data give support to the argument (Durand 2009) that for most speakers these stable initial schwas have to be considered as non-alternating mid front vowels. » (2013: 119). En effet, j'ai souvent insisté sur le fait que pour de nombreux locuteurs méridionaux conservateurs, les voyelles qu'on observe dans la pemière syllabe de genêt et jeunet ou fenêtre et feuillage sont strictement identiques du point de vue phonétique (un [ø] en accord avec la loi de position) et si la première n'alterne pas avec zéro, il n'y a aucune raison de la traiter comme un schwa phonologique (voir Durand, Slater et Wise 1987). Or, dans nos enquêtes méridionales, c'est effectivement ce que nous observons. Nous présentons ci-dessous un exemple typique de réalisation des séquences des jeunets et des genêts dans la liste de mots PFC. 


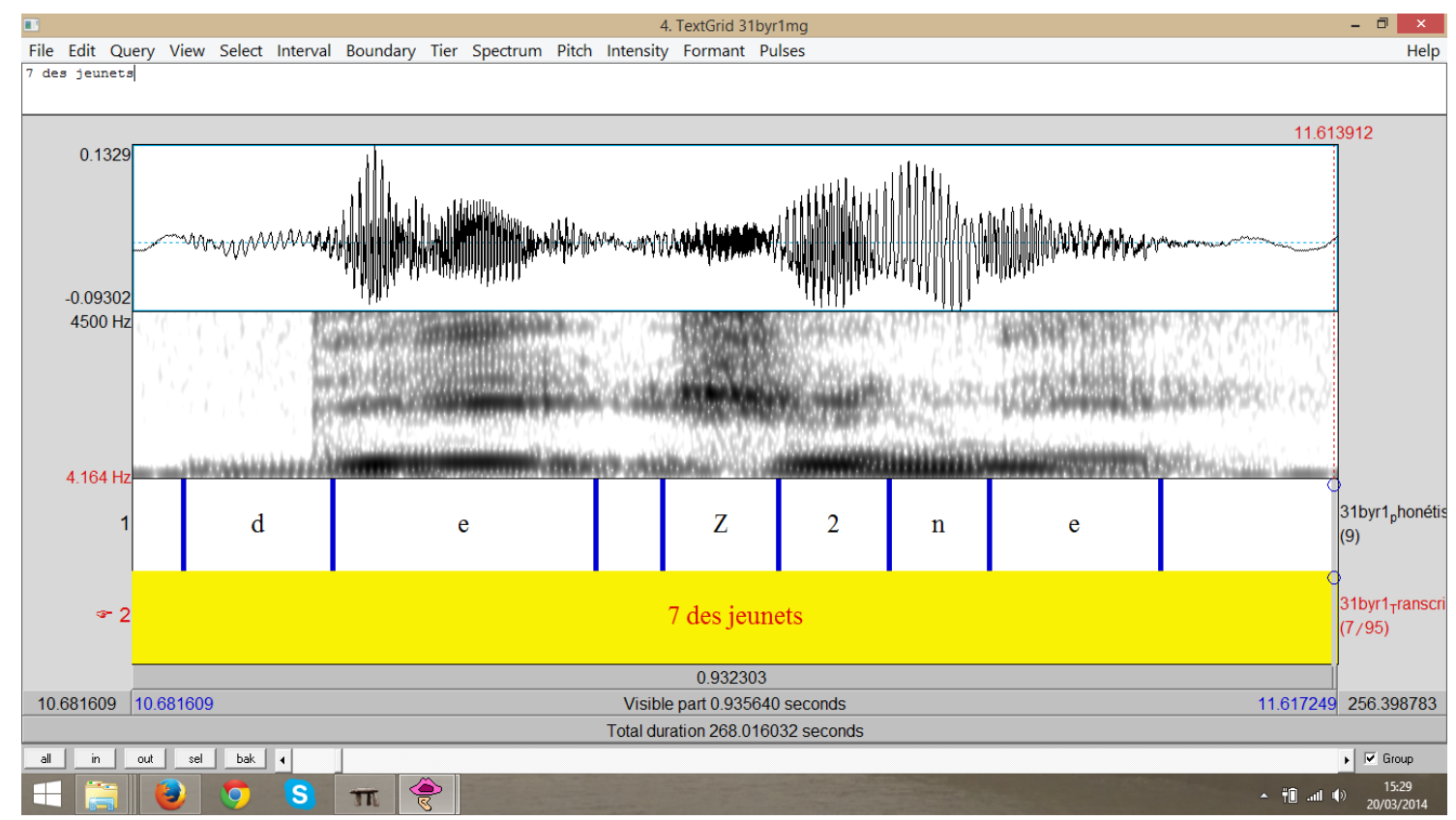

Figure 1 : Image spectrographique sous Praat de la séquence des jeunets par un locuteur de la sous-base Toulouse en lecture à haute voix de la liste de mots PFC.

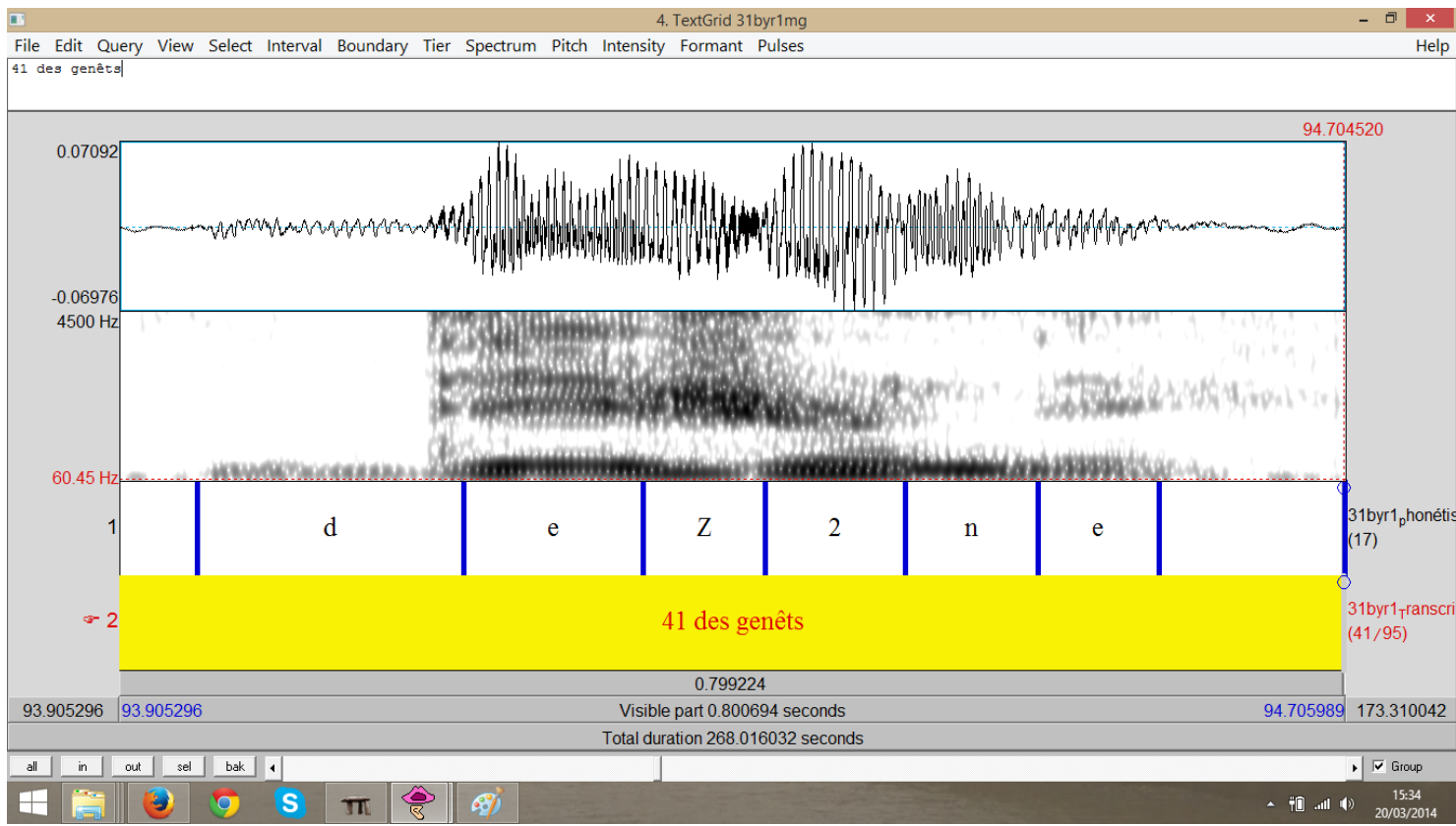

Figure 2 : Image spectrographique sous Praat de la séquence des genêts par un locuteur de la sous-base Toulouse en lecture à haute voix de la liste de mots PFC.

L'analyse des spectrogrammes en question démontre la quasi-identité acoustique de la première voyelle de jeunets et de celle de genêts avec les valeurs formatiques suivantes : F1 300 Hz, F2 1700 Hz, F3 2200 $\mathrm{Hz}$ pour jeunets et F1 $340 \mathrm{~Hz}, \mathrm{~F} 21800 \mathrm{~Hz}$ et F3 $2200 \mathrm{~Hz}$ pour genêts. Si on rapporte ces mesures aux 
moyennes formantiques que fournit Calliope (1989) on voit que ces voyelles sont à rattacher à un [ø] prototypique par opposition à [œ] ou un [ə].

(2) Valeurs formantiques typiques pour les voyelles du français selon Calliope (1989):

[ø]

$\mathrm{F} 1: 380 \mathrm{~Hz}$

$\mathrm{F} 2: 1400 \mathrm{~Hz}$

$\mathrm{F} 3: 2250 \mathrm{~Hz}$ [œ]

$\mathrm{F} 1: 550 \mathrm{~Hz}$

$\mathrm{F} 2: 1400 \mathrm{~Hz}$

F3 $: 2400 \mathrm{~Hz}$ [ə]

F1 : $450 \mathrm{~Hz}$

$\mathrm{F} 2: 1400 \mathrm{~Hz}$

$\mathrm{F} 3: 2100 \mathrm{~Hz}$

Ces observations justifient une remarque méthodologique qui me semble importante. À strictement parler, les codages PFC, que nous appelons par raccourci «codages schwa », ne démontrent pas que des schwas sont présents / absents dans tel ou tel contexte. Nos résultats sont chaque fois en attente d'une analyse phonologique et phonétique car les codages indiquent simplement qu'une voyelle est présente ou absente dans tel ou tel contexte pour laquelle la tradition réserve le terme 'schwa' (ou 'e muet' pour nous en tenir à ces deux étiquettes). Le statut de l'objet en question doit être établi par un raisonnement qui ne conduit pas nécessairement aux mêmes conclusions selon les variétés étudiées.

Si on revient maintenant aux exemples où un schwa putatif n'est pas prononcé dans les syllabes initiales par les jeunes générations on fait les constatations suivantes. Nous l'avons vu pour Aix-Marseille, ce phénomène affecte un petit nombre de mots et ces derniers sont très fréquents. Dans l'enquête LVTI à Toulouse, les 47 items où une voyelle n'est pas réalisée dans cette position portent uniquement sur des mots à forte fréquence du lexique français :

\begin{tabular}{|l|r|}
\hline & $\mathrm{n}=$ \\
\hline petit & 15 \\
\hline être (futur) & 12 \\
\hline re (répétition) + V & 4 \\
\hline semaine & 4 \\
\hline ferait/faisais & 3 \\
\hline demi & 2 \\
\hline demander & 1 \\
\hline venir & 1 \\
\hline regarder & 1 \\
\hline
\end{tabular}

Tableau 4. Absence de schwa en position initiale en fonction du lexique (données conversationnelles de l'enquête de Toulouse).

Comme le fait remarquer Eychenne (2009: 252) dans son étude du Pays-Basque, l'importance de la fréquence nous oriente vers des cadres comme celui de Bybee (2001), qui privilégient les usages. C'est le type d'approche qu'a adopté Pustka (2007) pour décrire diverses variétés du français au sein du programme PFC. Il est fort possible que, dans la phase actuelle d'évolution des accents méridionaux, les lexèmes concernés soient encodés de façon holistique avec deux formes liées à des contextes d'usage (par exemple /pti/ et /pøti/ pour petit) sans qu'il soit justifié de poser un mécanisme de non-correspondance entre des représentations phonologiques et des représentations phonétiques pour des exemples comme ceux du Tableau 4. Lorsque je parle d'effacement pour couvrir tous les cas de non-présence, c'est par 
commodité terminologique afin de ne pas alourdir une présentation donnant la priorité à la description plutôt qu'à une modélisation poussée.

Quel que soit le type d'analyse, les observations sur les variétés méridionales offrent un continuum qui part de lois « néogrammairiennes » du type

(3) $/ \mathrm{a} /-->\varnothing /-(\#) \mathrm{V}$

et passe par des effacements à la finale de mot qui ne sont pas liés à l'identité lexicale du mot (contexte $\mathrm{VCe} \# \mathrm{C}$ ou VCe\#\#) pour arriver à des alternances affectant les syllabes initiales de quelques mots fréquents (semaine / s(e)maine). J'ai défendu avec d'autres l'idée que les changements en cours s'appuyaient fortement sur la structure phonologique de ces variétés méridionales puisque la préférence à l'effacement qu'on peut symboliser de la façon suivante :

(4) Hiérachie des « effacements » : Syllabe finale > Syllabe interne > Syllabe initiale

coïncide dans les accents conservateurs avec la présence de vrais schwas phonologiques à la finale opposée à leur absence en syllabe initiale avec des entités au statut fluctuant en syllabe interne (vrai ou faux schwa ?). Je reprocherai donc à certaines études sociolinguistiques de ne pas vraiment examiner le statut linguistique des objets étudiés. Labov $(1972: 11)$ reprochait à juste titre à la linguistique classique de simplifier et de fausser les situations en construisant bien trop vite des formes phonologiques sousjacentes (/a/ entre barres obliques dans notre cas) et il recommandait la mise en place plus ouverte de variables entre parenthèses ((ə) en ce qui nous concerne ici). Mais attention le (ə) sans garde-fous qu'on trouve dans certains travaux sociolinguistiques est aussi problématique que le /o/ de la tradition phonologique. En tout état de cause, de grands changements sont en cours dans les variétés du français méridional. Ils n'affectent pas simplement des cibles phonologiques faibles. Ils remanient aussi certaines positions item par item, et nous forcent à repenser l'outillage conceptuel qui est nécessaire pour modéliser les fluctuations inter-variétés, inter-locuteurs et intra-locuteurs. Bien sûr, ces changements exigent aussi une interprétation sociologique. Comme le souligne Labov (1973:3) « one cannot understand the development of language change apart from the social life of the community in which it occurs. Or to put it another way social pressures are continually operating upon language not from some remote point in the past, but as an immanent social force acting in the living present. » Les facteurs sociopolitiques impliqués dans l'évolution des accents méridionaux à grande échelle sont très enchevêtrés et un ouvrage entier comme celui de Jones et Hornsby (2013) ne suffit pas pour examiner cette question dans toute sa complexité. Je me contenterai de signaler qu'il existe un état de subordination linguistique des variétés du Midi à l'égard des accents dits du nord de la Loire qui favorise le nivellement. Dans les enquêtes en cours à Toulouse, de nombreux locuteurs sont au mieux ambivalents quant aux mérites d'un accent dit de Toulouse, quand ils ne le trouvent pas archaïque, déshonorant ou vulgaire (Courdès-Murphy 2014). La prononciation d'un schwa appuyé en finale de mot est vue comme déclassante par de nombreux locuteurs méridionaux. Il constitue un des «shibboleths» de la prononciation du Midi avec des imitations du type « dans le Sud[ə] », qui sont à l'opposé de ce qu'on observe traditionnellement. ${ }^{9}$

\section{Le schwa en français de Suisse romande}

Certains des éléments évoqués dans l'analyse des variétés méridionales sont susceptibles de trouver un éclaircissement dans les données des points d'enquête PFC de Suisse romande (où trois points d'enquête PFC sont achevés - Nyon, Neuchâtel et Genève - et un est en cours de vérification - Martigny), 
notamment en ce qui concerne la syllabe initiale de mot. En effet, si, au niveau de l'inventaire phonologique, les variétés romandes ne diffèrent que très peu des variétés hexagonales septentrionales (Andreassen, Maître et Racine 2010, Racine et Andreassen 2012, Racine, Andreassen et Benetti, à paraître), elles sont souvent citées comme comportant un taux plus élevé de chute du schwa en syllabe initiale de polysyllabes (Walter 1982). Il est vrai qu'en Suisse romande, des occurrences telles que une s'maine ou quat(r)' ch'mises ou encore une f'melle sont tout à fait normales et fréquentes, alors qu'elles posent problème à toute théorie phonologique qui, directement ou indirectement, réfère les effacements à la bonne formation des groupes consonantiques attestés à l'initiale des mots. Si l'on suit cette hypothèse, on peut supposer que les données romandes fournissent un cadre adéquat pour approfondir la question de la chute du schwa dans cette position - position dans laquelle, on le rappelle, le schwa a tendance à se maintenir dans les variétés méridionales, excepté pour des mots extrêmement fréquents tels que petit ou semaine - et plus précisément celle du rôle de la fréquence lexicale sur le maintien ou la chute du schwa, dont le pouvoir explicatif a déjà été évoqué à de nombreuses reprises (Racine et Grosjean 2002, Pustka 2007, Racine 2008, Eychenne 2009) et qui est au cœur de l'approche de Bybee (2001), centrée sur les usages.

Un autre point sur lequel les données suisses romandes apportent des éléments intéressants concerne la nature phonétique de cette voyelle. En effet, comme nous le relevions précédemment, les codages schwa élaborés dans le cadre de PFC ne fournissent aucune indication sur la nature phonétique de la voyelle concernée. Des études acoustiques plus poussées sont par conséquent nécessaires afin d'explorer cette question. Pour de nombreux locuteurs méridionaux, nous venons de souligner que les voyelles observées dans la première syllabe de genêt et jeunet ou fenêtre et feuillage ne semblent pas différer en termes qualitatifs et qu'elles n'alternent pas avec zéro. Il n'y a donc aucune raison de les scinder en deux classes : schwa phonologique opposé à voyelle antérieure arrondie (Durand, Slater et Wise 1987). En revanche, nous avons rappelé que, pour certains spécialistes comme Walter (1976), la question du statut phonologique du «e muet» ne se pose en effet que pour les locuteurs qui possèdent un vrai schwa phonétique, qualitativement distinct de [ø] et de [œ]. Une analyse acoustique comparative effectuée sur les données PFC de trois points d'enquête, Brunoy, Québec et Nyon, semblent montrer que cela soit le cas pour les locuteurs suisses romands.

Dans ce qui suit, nous aborderons en premier lieu la question de la qualité vocalique du schwa suisse romand et nous enchaînerons avec une analyse des alternances observées dans les variétés romandes en position initiale de mots polysyllabiques en lien avec la fréquence lexicale et celle, estimée, des deux variantes de prononciation (semaine et s'maine). Nous terminerons en présentant des données récentes qui montrent l'existence, dans certaines variétés romandes, d'un lien entre la durée du schwa et son taux de maintien, données qui questionnent à la fois les théories phonologiques et les modèles psycholinguistiques de perception et production de la parole.

Notre bref survol du schwa suisse romand commencera donc par la question de sa qualité vocalique en syllabe initiale (chemise, pẹtit) et médiane (indiqueraient, dégeler). Bürki et al. (2008) ont comparé les réalisations du schwa dans la liste de mots et le texte lu de trois points d'enquête PFC différents, Brunoy (Ile-de-France), Québec (ville) et Nyon (Suisse romande). Dans cette recherche, les schwas de syllabe initiale et médiane de 9 mots présentant une alternance dans les données (présence/absence du schwa, 294 occurrences dont 150 produites avec schwa) ont été comparés aux réalisations des voyelles suivantes /i, u, $\mathrm{a}, \propto, \varnothing /$. Les résultats montrent d'une part qu'à Nyon, la qualité phonétique de la voyelle de ces mots diffère de celle de mots tels que neuf ou deux. Le schwa présente en effet une aperture intermédiaire à [œ, $\varnothing]$, tout en étant moins antérieur que celles-ci. Au niveau de l'arrondissement, il est plus proche de [œ] 
que de [ø]. Nos données montrent d'autre part qu'il se distingue du schwa de Brunoy, celui-ci étant plus fermé, tout comme semble l'être l'ensemble du système vocalique de cette variété d'Ile-de-France, par rapport aux deux autres régions. Ainsi, le schwa des locuteurs nyonnais se différencie donc à la fois de celui des deux autres variétés et des deux autres voyelles moyennes antérieures [œ] et [ø]. Walter (1976) restreint l'hypothèse d'un schwa phonologique à des éléments qui sont aussi des schwas phonétiques comme dans les variétés de Suisse romande que nous examinons ici, mais c'est à mes yeux une position bien trop restrictive. Revenons donc succinctement sur la question phonologique des alternances en syllabe initiale.

La première constatation qui s'impose est qu'il est nécessaire, pour cette variété comme pour de nombreuses variétés du français hexagonal, de postuler une division en trois classes de mots : i) les mots de type \#CC (ex. ski, smala) qui ne présente pas d'alternance; ii) les mots de type \#C(ə)C (ex. s(e)couer, $s(e) m a i n e)$; iii) les mots \#CVC, avec une voyelle qui n'alterne jamais et qui peut être soit mi-fermée (ex. jeunet réalisé $[\zeta ø n \varepsilon]$ dans les variétés romandes) soit mi-ouverte (ex. feuillage réalisé [fœjaz]). Les lexèmes qu'il faut rattacher à telle ou telle classe varient légèrement de système à système mais la tripartition est bien établie. Si l'on se réfère aux différentes positions théoriques présentées en ouverture de ce chapitre, cette division, si elle s'oppose à la posture défendue par Martinet, rejoint en revanche celle adoptée par la tradition générative et le travail minutieux de Dell (1973). En effet, la solution épenthétique que prônait Martinet se heurte au fait que les mots commençant par des groupes de consonnes stables forment un sous-ensemble respectable et en constante augmentation en français (par exemple, smicard face à s(e)maine en français hexagonal). Formuler des règles d'insertion de schwa qui ne soient pas arbitraires et ne surgénèrent pas n'a jamais été fait de façon convaincante. Il faut donc donc distinguer phonologiquement les mots à initiale $\# \mathrm{CC}(\mathrm{C})$ des mots qui alternent entre présence et absence et des mots où la voyelle est stable (par exemple, gueulard). Une fois de plus, il semble important de souligner que la graphie ne constitue pas une preuve indubitable de l'appartenance d'un mot à une des trois classes défendues ici. Pour beaucoup de locuteurs, les mots belette ou belon ont une voyelle antérieure arrondie stable qui n'est pas un schwa. On parle parfois de «schwa stabilisé » pour de tels mots. Si l'analyse diachronique peut démontrer que la première voyelle de ces mots était véritablement un schwa phonologique et phonétique dans une période antérieure au présent, le terme de schwa stabilisé paraît adéquat. En tout état de cause, je défendrai l'idée qu'il faut bien poser une place phonologique pour les éléments qui alternent entre présence et absence, quelle que soit leur qualité phonétique dans les réalisations. Andreassen (2013) démontre que, psycholinguistiquement, l'enfant privilégie au départ la présence d'un élément qui se sépare en deux sous-classes (alternante vs. stable) dans le système adulte.

Penchons-nous maintenant sur la deuxième classe de mots, celle qui présente des alternances présence/absence et possède de surcroît un «vrai » schwa phonétique dans les variétés suisses étudiées ici. Cette position semble très dynamique dans les variétés romandes puisque l'on peut fréquemment y observer des formes qui, si elles sont absentes des variétés méridionales, peuvent certes apparaître dans les variétés septentrionales hexagonales mais de manière beaucoup moins systématique. Les études de Racine et Grosjean (2002), Racine (2007) et Racine (2008) montrent que, comme pour les données méridionales, la fréquence lexicale des mots en Suisse romande est une variable importante (ex. schwa davantage maintenu dans dẹmeure que dans dẹmande), de même que la fréquence estimée de production des deux variantes, avec et sans schwa. Ces deux facteurs semblent d'ailleurs constituer de meilleurs prédicteurs des productions des locuteurs que le contexte segmental environnant. Au niveau de la fréquence estimée des variantes, les données de Racine (2008) montrent un décalage dans les jugements français (12 locuteurs de la région de Nantes) et suisses (12 locuteurs de la région de Neuchâtel) devant 
les deux formes (avec et sans schwa) de 378 substantifs comportant un schwa en syllabe initiale (ex. la semaine). Les jugements ne sont pas fondamentalement différents mais plutôt « décalés ». Les Suisses romands acceptent en effet mieux l'effacement du schwa dans ce type de mots que les Français, avec toutefois des différentes parfois très saillantes pour certains mots. Ainsi, pour le mot femelle, l'alternance (fémelle/f'melle) est tout à fait attestée à Neuchâtel - et de manière générale en Suisse romande - alors que cela ne semble pas du tout être le cas à Nantes.

Nous conclurons cet examen du schwa suisse en abordant la question de sa durée en position initiale. Des données antérieures de Racine et Grosjean (2002) avaient révélé l'existence d'une corrélation entre le taux de maintien du schwa et sa durée : pour un mot donné, plus le taux de maintien est élevé, plus le schwa, lorsqu'il est produit, est long. Ainsi, pour le mot menu, le taux de maintien dans leur étude de production était de $81.25 \%$ et la durée moyenne du schwa - lorsqu'il était présent - représentait $20.29 \%$ de la durée totale du mot. Pour le mot repassage, en revanche, le taux de maintien n'était que de 26.67\% et la durée du schwa ne représentait que $7.42 \%$ de la durée totale du mot. L'hypothèse évoquée était celle d'un processus continu de compression de la voyelle, qui aboutirait, à un stade ultime, à la suppression complète de celle-ci (cf. également Bürki et al., 2007 et, pour des données similaires pour l'anglais, cf. Patterson et al., 2003). Le travail récent d'Andreassen et Racine (2013) nous permet de franchir une étape supplémentaire. Ces chercheuses montrent que, si cette corrélation existe, elle semble modulée par des différences entre les régions. Une étude comparative menée sur les données PFC des deux conversations des points de Neuchâtel, Nyon et Martigny révèle en effet des différences régionales importantes. Les données neuchâteloises présentent un taux de maintien significativement moins élevé que celui des deux autres régions, tout en se différenciant de ces dernières également par la durée du schwa, qui est plus long dans cette variété. Les locuteurs vaudois et valaisans semblent donc maintenir davantage le schwa que les Neuchâtelois, mais avec des schwas plus variables, qui peuvent parfois être très courts. Ces résultats récents interrogent l'analyse phonologique traditionnelle du schwa, limité à un processus catégoriel (présence $v s$ absence). En outre, au niveau psycholinguistique, ils apportent des données intéressantes dans le débat qui oppose les tenants de l'approche abstractionniste à ceux de l'approche exemplariste, en favorisant plutôt la deuxième approche ou une approche intermédiaire, très en vogue actuellement, celle des modèles hybrides (Nguyen, Wauquier et Tuller, 2009).

\section{Conclusion}

$\mathrm{Au}$ terme de ce parcours, qu'on me permette d'être bref. Je soulignerai tout simplement que la méthodologie PFC, en ce qui concerne le schwa, offre une approche collaborative qui permet de renouveler les données dans la diversité des usages, de les explorer sous divers angles et de modéliser les systèmes dans leur dynamique interne. En refusant de placer notre travail au sein de cases étanches (phonétique, phonologie, dialectologie, sociolinguistique, psycholinguistique), nous pensons contribuer à la construction d'une véritable linguistique de l'oral. C'est donc un programme que nous défendons et non un modèle verrouillé soucieux de la seule survie de ses principes théoriques. Libre au lecteur intéressé de rejoindre ou non ce programme collaboratif, d'en utiliser ou non les résultats. Les enquêtes à réaliser restent nombreuses et le traitement des enquêtes disponibles exige des perspectives croisées pour en tirer la «substantifique moëlle ». La comparabilité des données qu'autorise notre méthode d'enquête reste un atout important de notre approche. Élargissons sans crainte nos études vers des enquêtes plus «écologiques » sur les variétés du français mais ne perdons pas de vue que cet objet protéiforme et apparemment sans voix qu'est le « e muet » est susceptible de nous interpeller au détour de nos analyses. 


\section{Références bibliographiques}

Anderson, S.R. (1982) The analysis of French schwas: or, how to get something out of nothing. Language $58,535-571$.

Andreassen, H.N. (2013). Distribution and Acquisition in the Light of Swiss French Data. Thèse de doctorat. Université de Tromsø.

Andreassen, H. N. et Eychenne, J. (2013). The French Foot Revisited. Language Sciences 39, 126-140

Andreassen, H., Maître, R. et Racine, I. (2010). La Suisse. Dans S. Detey, J. Durand, B. Laks et C. Lyche (éds). Les variétés du français parlé dans l'espace francophone: ressources pour l'enseignement. Paris : Ophrys, 211-233.

Andreassen, H. N. et Racine, I. (2013). Schwa et variation inter-régionale : une analyse de trois points d'enquête suisses. Journées PFC 2013 «Regards croisés sur les corpus oraux », Paris, 5-7 décembre 2013.

Arnauld, A. et C. Lancelot (1660). Grammaire générale et raisonnée. Réimprimé en 1969 avec une préface de Michel Foucault. Paris : Republications Paulet.

Bourdieu, P. (2004). Esquisse pour une auto-analyse. Paris : Éditions Raisons d’Agir.

Bürki, A., Fougeron, C., Gendrot, C. \& Frauenfelder, U. (2007). De l'ambiguïté de la chute du schwa en français. Schwa(s). Actes des $5^{\text {emes }}$ Journées d'études linguistiques 2007, Université de Nantes, 27-28 juin $2007,83-88$.

Bürki, A., Racine, I., Andreassen, H., Fougeron \& C., Frauenfelder, U. (2008). Timbre du schwa en français et variation régionale: une étude comparative. Actes des 27èmes Journées d'études sur la parole, juin 2008, Avignon.

Bybee, (2001). Phonology and Language Use. Cambridge : Cambridge University Press.

Calliope (1989), La parole et son traitement automatique. Paris : Masson

Côté, M.-H. (2000). Consonant Cluster Phonotactics : a Perceptual Approach. Thèse de doctorat. MIT.

Côté, M.-H. (2008). Empty elements in schwa, liaison and h-aspiré: The French Holy Trinity reconsidered. In J. Hartmann, V. Hegedüs et H. van Riemsdijk (eds) Sounds of Silence : Empty Elements in Syntax and Phonology. Amsterdam : Elsevier, 61-103.

Côté, M.-H. (2009). Contraintes segmentales et variation dans la perte et la stabilisation du schwa en syllabe initiale. In L. Baronian et F. Martineau (eds), Le français d'un continent à l'autre. Mélanges offerts à Yves-Charles Morin. Québec : Presses de l'Université Laval, 93-121.

Côté, M.-H. (2012). Laurentian French (Quebec): extra vowels, missing schwas and surprising liaison consonants. In R. Gess, C. Lyche, and T. Meisenburg (eds.). Phonological Variation in French: Illustrations from three continents. Amsterdam/Philadelphia: John Benjamins, 235-274. 
Courdès-Murphy, L. (2013). Contribution à l'étude de la phonologie du français contemporain: Le français parlé à Toulouse. Mémoire de M1. Université de Toulouse 2 Le Mirail.

Courdès-Murphy, L. (2014). Les voyelles nasales en français de Toulouse: empirie, modélisation et dynamique des systèmes. Mémoire de M2. Université de Toulouse 2 Le Mirail.

Delattre, P. (1951). Principe de phonétique française. Middlebury, Vermont : Middlebury College.

Dell, F. (1973a). Les règles et les sons. Introduction à la phonologie générative. Paris : Hermann.

Dell, F. (1973b). E muet. Fiction graphique ou réalité orthographique. In S. Anderson et P. Kiparsky (eds) A Festschrift for Morris Halle. New York : Holt, Rinehart and Winston, 26-50.

Dell, F. (1985). Les règles et les sons. Introduction à la phonologie générative. Deuxième édition révisée. Paris : Hermann.

Detey, S., Durand, J., Laks, B. and Lyche, C. (eds.)(2010). Les variétés du français parlé dans l'espace francophone. Ressources pour l'enseignement. Paris: Ophrys.

Durand, J. (1976). Generative Phonology, Dependency Phonology and Southern French. Lingua e Stile $11,3-23$.

Durand, J. (986) French liaison, floating segments and other segments in a dependency framework. In J. Durand (ed) Dependency and Non-Linear Phonology. Londres : Croom Helm, 161-201.

Durand, J. (2009). Essai de panorama phonologique : les accents du midi. In L. Baronian et F. Martineau (eds), Le français d'un continent à l'autre. Mélanges offerts à Yves-Charles Morin. Québec : Presses de l'Université Laval, 123-170.

Durand, J. et Eychenne, J. (2004). Le schwa en français. Pourquoi des corpus ? Corpus 3, 311-356.

Durand, J., Eychenne, J., Lyche, C. (2013). On levelling and counter-levelling in French: a phonological perspective. ». In M. C. Jones et Hornsby (eds), Language and Social Structure in Urban France. Leeds : Maney.

Durand, J., Laks, B., Calderone, B. et Tchobanov, A. (2011). Que savons-nous de la liaison aujourd'hui ? Langue Française 169: 103-126.

Durand, J., B. Laks et C. Lyche (2003a). Le projet (Phonologie du français contemporain) PFC. In J. Durand, B. Laks et C. Lyche (eds) Le français dans sa variation. La Tribune internationale des langues vivantes $33,3-9$.

Durand, J., B. Laks et C. Lyche (eds)(2003b). Le français dans sa variation. La Tribune internationale des langues vivantes, vol. 33 .

Durand, J., Laks, B. et Lyche, C. (2009). Le projet PFC (Phonologie du français contemporain): une source de données primaires structurées. In J. Durand, B. Laks et C. Lyche (eds), Phonologie, variation et accents du français. Paris : Hermès, 19-62. 
Durand, J., B. Laks et C. Lyche (eds)(2009b). Phonologie, variation et accents du français. Paris : Hermès.

Durand, J., B. Laks et C. Lyche (2014). French Phonology from a Corpus Perspective : the PFC programme. In J. Durand, U. Gut et G. Kristoffersen (eds), The Oxford Handbook of Corpus Phonology. Oxford : Oxford University Press, 486-497.

Durand, J. and Przewozny, A. (2012). La phonologie de l'anglais contemporain : usages, variétés et structure. Revue française de linguistique appliquée 17(1): 25-36.

Durand, J., Slater, C. et Wise, H. (1987). Observations on Schwa in Southern French. Linguistics 25/2, 983-1004.

Encrevé, P. (1988). La liaison avec ou sans enchaînement. Phonologie tridimensionnelle et usages du français. Paris : Seuil.

Eychenne, J. (2006). Aspects de la phonologie du schwa en français contemporain. Optimalité, visibilité prosodique, gradience. Ph.D. dissertation, University of Toulouse-Le Mirail.

Fitch, W.T., M.D. Hauser et N. Chomsky (2005). The Evolution of the Language Faculty: Clarifications and implications. Cognition 97, 179-210.

Fouché, P. (1956). Traité de prononciation française. Paris : Klincksieck.

Gess, R., C. Lyche et T. Meisenburg (eds) (2012). Phonological Variation in French : Illustrations from Three Continents. La Haye : Benjamins.

Grammont, M. (1894). Le patois de la Franche-Montagne et en particulier de Damprichard (FrancheComté). IV : La loi des trois consonnes. Mémoires de la Société de Linguistique de Paris 8 : 53-90.

Grevisse, M. (1964). Le bon usage. (8ème édition.) Gembloux, Duculot.

Hansen, A.B. (2003). Le contexte prépausal - un contexte dynamique pour le schwa dans le français parisien. In J. Durand, B. Laks \& C. Lyche (eds), La prononciation du français dans sa variation, La tribune internationale des langues vivantes, no. 33, 142-144.

Hansen, A.B. (2012). A Study of Young Parisian Speech : Some Trends in Pronunciation. In R. Gess, C. Lyche, and T. Meisenburg (eds.), Phonological Variation in French: Illustrations from three continents. Amsterdam/Philadelphia: John Benjamins, 151-172.

Keller, F. (2006). Linear optimality theory as a model of gradience in grammar. In G. Fansebas, C. Féry, R. Vogel et M. Schlesewski (eds), Gradience in Grammar: Generative Perspectives. Oxford: Oxford University Press, 270-287.

Labov, W. (1972). Sociolinguistic Patterns. Oxford : Basil Blackwell.

Laks, B. (2002). Description de l'oral et variation: La phonologie et la norme. L'information grammaticale 94: 5-11. 
Laks, B., Calderone, B. and Chelata, C. (2014). French liaison and the lexical repository. In C. Chelata and S. Calamai (eds). Advances in Sociophonetics. Amsterdam: Benjamins, 29-54.

Lyche, C. (1978). Glides in French: Questions for Natural Generative Phonology.' Lingua 49, 315-330.

Lyche, C. (à paraître). The Schwa Level. In S. Detey, J. Durand, B. Laks et C. Lyche (éds), Varieties of Spoken French : a source book. Oxford : Oxford University Press.

Martinet, A. (1945). La prononciation du français contemporain. Genève : Droz.

Martinet, A. (1969). Le français sans fard. Paris : Presses Universitaires de France.

Martinet, A. et Walter, H. (1973). Dictionnaire de la prononciation française dans son usage réel. Paris : France-Expansion.

Martinet, A. et Walter, H. (1973). Dictionnaire de la prononciation française dans son usage réel. Paris : France-Expansion.

Morin, Y.-C. (1978). The status of mute 'e'. Studies in French Linguistics 1, 79-140.

Morin, Y.-C. (1987). Quelques observations sur la chute du e muet dans le français régional de SaintEtienne. La linguistique 29(1), 71-93.

Nguyen N. and Adda-Decker, M. (eds.)(2013). Analyse phonétique des grands corpus oraux: méthodes et outils. Paris : Hermès-Lavoisier.

Nguyen, N., Wauquier, S. \& Tuller, B. (2009). The dynamical approach to speech perception: from fine phonetic detail to abstract phonological categories. In F. Pellegrino, E. Marsico, I. Chitoran, \& C. Coupé (eds.), Approaches to Phonological Complexity. Berlin: Mouton de Gruyter, 193-217.

Patterson, D., LoCasto, P. C. \& Connine, C. M. (2003). Corpora analyses of frequency deletion in conversational American English. Phonetica 60, 45-68.

Popper, K. (2004). Conjectures and refutations : the growth of scientific knowledge (Reprinted. ed.). London: Routledge.

Pustka, E. (2007) Phonologie et variétés en contact. Aveyronnais et Guadeloupéens à Paris. Tübingen: Narr.

Racine, I. (2007). Effacement du schwa dans des mots lexicaux : constitution d'une base de données et analyse comparative. Schwa(s). Actes des 5èmes des Journées d'études linguistiques 2007, Université de Nantes, 27-28 juin 2007, 125-130.

Racine, I. (2008). Les effets de l'effacement du schwa sur la production et la perception de la parole en français. Thèse de doctorat, Université de Genève. 
Racine I., Andreassen, H. (2012). A phonological study of a Swiss French variety : data from the Canton of Neuchâtel. In R. Gess, C. Lyche et T. Meisenburg (eds), Phonological Variation in French : Illustrations from Three Continents. Amsterdam : John Benjamins, 173-207.

Racine, I., Andreassen, H. \& Benetti, L. (à paraître). Swiss French. In S. Detey, J. Durand, B. Laks et C. Lyche (éds), Varieties of Spoken French : a source book. Oxford : Oxford University Press.

Racine, I. \& Grosjean, F. (2002) La production du E caduc facultatif est-elle prévisible? Un début de réponse. Journal of French Language Studies, 12 (3), 307-326.

Racine, I., Schwab, S. \& Detey, S. (2013). Accent(s) suisse(s) ou standard(s) suisse(s) ? Approche perceptive dans quatre régions de Suisse romande. Dans A. Falkert (éd.). La perception des accents du français hors de France. Mons : Editions CIPA, 41-59.

Ranson, D.L. et Passarello, M.D. (2012). L'élision variable du schwa en fin de mot chez des hommes méridionaux : L'effet des consonnes environnantes et de la fréquence de la lexie. In F. Neveu et alii. (2012) Actes du $3^{\text {ème }}$ Congrès Mondial de Linguistique Française (CMLF-2012), EDP Science, 15191535 .

Schane S.A. (1968a). French Phonology and Morphology. Cambridge, Mass. : M.I.T Press.

Schane, S.A. (1968b). On the abstract character of French « e muet ». Glossa 150-163.

Scheer, T. (1999). «Aspects de l'alternance schwa-zéro à la lumière de 'CVCV'». Recherches linguistiques de Vincennes $28: 87-114$.

Scheer, T. (2000). L’immunité de schwa en début de mot. Langue française 126 : 113-126.

Séguy, J. (1951). Le français parlé à Toulouse. Toulouse : Privat.

Simon, A.C. (ed.)(2012). La variation prosodique régionale en français. Bruxelles : De Boeck-Duculot.

Tranel, B. (1987). French schwa and non linear phonology. Linguistics 25, 845-866.

Tranel, B. (2000). Aspects de la phonologie du français et la théorie de l'optimalité. Langue Française $126,39-72$.

Walter, H. (1976). La dynamique des phonèmes dans le lexique français contemporain. Paris : FranceExpansion.

Walter, H. (1982). Enquête phonologique et variétés régionales du français. Paris: PUF.

\footnotetext{
${ }^{1}$ Je remercie infiniment Helene Andreassen, Léa Courdès-Murphy, Sylvain Detey, Julien Eychenne, Chantal Lyche et Rudolph Sock pour leur aide, les données partagées et leurs recommandations dans la préparation de ce travail.
} 
Isabelle Racine m'a prodigué de très nombreux conseils et je lui suis redevable de cetaines idées exprimées ici. Je demeure seul responsable des interprétations avancées et des erreurs probables que décèlera le lecteur attentif.

${ }^{2}$ Voir Durand, Laks et Lyche (2003a,b, 2009a,b, 2014), Detey, Durand, Laks et Lyche (2010), Gess, Lyche et Meisenburg (2013), et consulter le site : www.projet-pfc.net. Pour des développements autour de PFC, voir Nguyen et Adda-Decker (2013) et Simon (2012).

${ }^{3}$ Les analyses du schwa dans le cadre de PFC sont très nombreuses. La plupart des contributions aux volumes mentionnés à la note 2 contiennent des développements sur le schwa. Pour des travaux où le schwa est abordé de façon plus complète, on citera Durand et Eychenne (2004), Eychenne (2006), Pustka (2007), Côté (2012), Hansen (2012), Andreassen et Eychenne (2013), Lyche (à paraître). Pour la position finale de mot, l'étude de Ranson et Passarello (2012) est une étude fouillée du schwa dans les variétés méridionales. Ces auteurs, à partir de données diverses, dont des enquêtes PFC, montrent la complexité des facteurs phonétiques et fréquentiels qui favorisent l'effacement. La meilleure source de références sur le schwa actuellement disponible est Andreassen (2013), une des études les plus novatrices sur la question. Pour une étude remarquable du 'e' muet en français régional, voir Morin (1983).

${ }^{4} \mathrm{Ou}$ [pøtitanimal] ou [pətitanimal]. Le symbole ' $\leftrightarrow$ ' a malheureusement une double valeur dans les travaux spécialisés: vraie voyelle centrale ou représentation abstraite qui est indice de l'alternance entre voyelle et zéro.

5 Julien Eychenne me rappelle qu'il y a également beaucoup d'études psycholinguistiques montrant que les jugements grammaticaux sont souvent non catégoriques et que c'est un argument qui a été avancé en faveur des modèles probabilistes : voir, par exemple, Keller (2006).

${ }^{6}$ La question des positions vides et une discussion des modèles comme la théorie 'CVCV' dépassent les limites de cet article : voir Scheer $(1999,2000)$.

${ }^{7}$ Voir Courdès-Murphy $(2013,2014)$. Le projet LVTI est coordonné par Hélène Giraudo, Anne Przewozny, JeanMichel Tarrier et Jacques Durand. Il vise à une comparaison à grande échelle de Toulouse et Manchester. Pour une présentation rapide, voir Durand et Przewozny (2011).

${ }^{8}$ Nous utilisons le terme 'position initiale' de façon lâche puisque le schwa ne peut pas être en position initiale de mot mais doit être précédé d'une attaque consonantique. C'est un des arguments déployés par les partisans d'une position vide pour le schwa puisqu'une attaque vide plus un noyau vide donne une syllabe vide. Dans ce travail, 'position initiale' renvoie à un schwa putatif en syllabe initiale de mot.

${ }^{9}$ Dans ce contexte, les locuteurs ultra conservateurs en Languedoc, par exemple, dévoisent le /d/: [sut]. Le dévoisement des obstruantes en position finale de mot est désormais récessif dans nos enquêtes. 